\title{
Understanding Motivations and Player Experiences of Older Adults in Virtual Reality Training
}

Aijse W. de Vries ${ }^{1}$, Jaap H. van Dieën²; Vero van den Abeele ${ }^{3}$, Sabine M.P. Verschueren ${ }^{1}$

${ }^{1}$ Musculoskeletal Research Unit, Department of Rehabilitation Sciences, Faculty of Kinesiology and Rehabilitation Sciences, Katholieke Universiteit Leuven, Leuven, Belgium

${ }^{2}$ MOVE Research Institute Amsterdam, Faculty of Human Movement Sciences, VU University Amsterdam, Amsterdam, The Netherlands

${ }^{3}$ Computer Science Technology TC, Group T, Faculty of Engineering Technology, Katholieke Universiteit Leuven, Leuven, Belgium

e-mail:

Corresponding author: Prof. dr. Sabine M.P. Verschueren: sabine.verschueren@kuleuven.be

First author: Aijse de Vries: aijse.devries@kuleuven.be

\section{Acknowledgements}

This research was funded by the European Commission through MOVE-AGE, an Erasmus Mundus Joint Doctorate programme (2011-0015). 


\begin{abstract}
Objective: We studied which games and underlying game mechanics are considered motivating by older adults, so that designers and therapists make informed choices when designing or selecting VR-training interventions.
\end{abstract}

Materials and methods: We conducted a repeated measures design with 30 older participants, who played eight different VR-training games, and afterwards filled out the Intrinsic Motivation Inventory (IMI). Differences in intrinsic motivation between games were analyzed using Friedman's tests. In addition, in-depth interviews were conducted according to the laddering technique, to unveil the underlying game mechanics that lead to the players preferences.

Results: IMI scores were relatively high for all games, indicating that these VR games seem effective for inducing a high intrinsic motivation. Wii yoga and Kinect Adventures were the highest scoring games on all but the negative subscale tension. Both games provided regular positive feedback. An important game mechanic was Variation, which showed a strong link to important values such as: to Stay Focused, Improve Fitness and Health and Independency. Furthermore, the game mechanics Visual Feedback and Positive Feedback, which lead to an increased Drive to Perform, were perceived valuable. Seemingly contradicting, but both important attributes such as Speed versus Slow Movements, emphasize the importance of designing VR training that adapts to the skill level of the player.

Conclusion: We have shown that games with different game mechanics can induce high intrinsic motivation. When designing or selecting VR balance training games for older adults, these game mechanics should be incorporated to optimize a positive user experience and increase intrinsic motivation. 


\section{Introduction}

About $30-40 \%$ of the home dwelling adults aged above 65 , experience one or more falls a year and about $10-20 \%$ of these falls result in serious injury requiring hospitalization ${ }^{1,2}$. Virtual reality (VR) training for older adults to improve balance and prevent falls is an emerging field of study. ${ }^{3-5}$ An increase in motivation and adherence is mentioned as one of the possible benefits of VR training, ${ }^{6}$ as compared to more traditional training programs. Adherence is an important factor in balance training, since balance training effects diminish when the training program is ceased, ${ }^{7,8}$ However, adherence in home-based balance training programs for older adults is low. ${ }^{9}$ Low adherence might be averted through the motivational qualities of VR training. ${ }^{10,11}$

The term virtual reality covers a wide range of applications, ${ }^{12}$ in physical rehabilitation, computer games that are controlled with body movements are often referred to as exergames or VR training, the latter is the terminology adopted in this work.

While several studies assessed the usability of VR training, only few studies investigated the motivational affordances of VR-based training. For example a review by Nawaz et al., which focused on acceptability, concluded that VR games are received well by older adults. ${ }^{13}$ Furthermore, studies on the preferences of older gamers show that older gamers prefer casual games, such as puzzle games over so called hardcore games. ${ }^{14,15}$ However, the preferences of older adults, specifically for VR-training games are not yet extensively investigated, even though adjusting VR training to their preferences might increase intrinsic motivation. ${ }^{16}$ Intrinsic motivation is the motivation to participate in an intervention because the intervention in and of itself is motivating. ${ }^{17,18}$ Therefore, if we want to improve adherence in VR training, we should aim to incorporate game mechanics that optimize the intrinsic motivation of VR-training games. The Intrinsic Motivation Inventory (IMI), can be used to measure intrinsic motivation to perform a task. ${ }^{16,19}$ A study on VR-balance-training for older adults, showed higher IMI scores in the subscales enjoyment in an exergaming intervention compared to conventional training. ${ }^{20}$ Furthermore, intrinsic motivation has previously shown to be predictive of the time invested in playing a game. ${ }^{15}$

The relation between game mechanics and player motivations can be further investigated through the lens of Means-End Theory. Means-End Theory posits that consumers or players in this case, will prefer one game to another because of how certain attributes or game mechanics, lead to certain benefits, or desired consequences, or ultimately tailor to the goals or values a player seeks. Hence, the goal of the player is the end point of an action, the way to get there are the means. ${ }^{21,22}$ This approach thus allows 
studying which characteristics of the game (attributes) the individual considers important for what outcomes (consequences) and why these consequences matter to the individual (values).

User Experience Laddering (UX-Laddering) is a technique to reveal Means-End chain (MEC) structures. ${ }^{23}$ Via a semi-structured interview participants are asked to select their favorite choice, often via preference ranking. ${ }^{24}$ The interviewer will then derive the attributes, consequences and values underlying the choices of the participants, by means of in-depth interviews. UX-Laddering has previously been applied in Human-Computer Interaction $(\mathrm{HCl})$ and games research, for example to understand gaming preferences of preschoolers. ${ }^{24}$ However, it has not yet been applied to the field of VR balance training for older adults.

The aim of this study was to reveal which VR-training games older adults perceive as being intrinsically motivating, as measured by the IMI. Furthermore, we studied their preferences and the underlying Means-End structures, to uncover game mechanics that explain why certain VR-training games are more appealing to older adults.

\section{Methods}

\section{Participants}

We recruited 30 older adults (table 1), who reported to have no physical or cognitive diseases and could stand for at least 20 minutes, by distributing flyers at the sports facilities and social events for older adults. All participants were older than 65 , lived independently and scored above the inclusion threshold of 26 on the mini mental state exam (MMSE), ${ }^{25}$ All participants signed a written informed consent, in accordance with the declaration of Helsinki. The local ethics committee (Commissie Medische Ethiek K.U. Leuven) approved the study.

\section{Games}

Eight VR-training games, which were played on three different systems, Wii (Nintendo, Kyoto, Japan); Xbox 360 (Microsoft, Redmond, WA, US) and Dynstable (MotekForcelink, Amsterdam, Netherlands), were chosen for this study. Games were selected to represent a varied but realistic sample of games, to be used in VR training. The Wii and Xbox are both of the shelf gaming systems, whereas Dynstable is developed for the rehabilitation market. The games we selected and the abbreviations we used are listed in table 2. A brief description of the included games is included in the Appendices. 
Protocol

The order of the games was determined by a computer-based random number generator. ${ }^{26}$ Participants played each game three times, except for the Kinyoga and Boxing games, which were longer games that already incorporated repeating sequences. IMI questionnaires were filled out in Access (Microsoft, Redmond, USA), directly after playing each game. After all games were played, we commenced the UXladdering interview.

\section{IMI Analysis}

The questions of the IMI questionnaire are categorized to form seven different subscales from which the relevant subscales can be included in the study. ${ }^{19}$ We excluded the subscales perceived choice, because the game selection was predetermined, and relatedness, because this subscale measures how well the person can relate to the character in the game. Most of the included games did not involve such a character. We included the following subscales: enjoyment, competence, effort, value, and tension, of which tension is a negative trait and enjoyment can be considered as a self-report of intrinsic motivation. ${ }^{19}$ For all subscales, Cronbach's alphas were relatively high (table 2), indicating internal consistency between the items that constitute each subscale.

To test the hypothesis that different games elicit different levels of intrinsic motivation, we choose Friedman's tests, because the data was not normally distributed. The ranking and further statistical analysis was performed in IBM SPSS Statistics Version 21.0. To reduce the amount of comparisons, posthoc analysis was done using stepwise step-down analysis, in which homogeneous conditions are clustered. 


\section{UX-laddering interview}

In the UX-laddering interview, we presented the participants with screen captures of the games they played, in pairs of two, according to a preference ranking method, shown previously to be effective for laddering studies (fig. 1). ${ }^{24}$ The order was the same as the order in which they played the games. For each pair, participants were asked to identify their preferred game, which started the semi-structured interview in which the interviewer probed the participant with the question 'why' in an attempt to reveal the UX ladder. The participant was asked why they preferred this game, this usually leads to the consequences that drew the participant towards their preferred game. This question was followed by the question what aspect in the game caused this consequence, to reveal the preferred attributes. The next question, why the mentioned consequence is important to them, aimed to reveal the underlying personal values of the participant. The responses were noted down, to be coded and analyzed. We also kept a record of the preferred games, their second and favorite game would receive one and two points respectively.

\section{Analyzing the UX-laddering interviews}

Two independent coders translated the interviews into key terms or core elements, which formed the elements of the individual ladders. The ladders from both coders were entered in the UX-laddering software. ${ }^{27}$ These ladders form the implication matrix, where for each core element it is charted how many times it has been linked to another core element. From the implication matrix the Hierarchical Value Map (HVM) is created. The HVM is a web shaped figure, which graphically visualizes the connections between the core elements. Cut-off values had to be established, for the minimal number of connections between core elements that would be represented on the HVM. Even though the literature recommends to use cut off values that maintain about two thirds of all links in the HVM, ${ }^{23}$ we set stricter cut-off values, to achieve a stable yet clear HVM, which maintained $40 \%$ of all linkages.

\section{Results}

\section{Quantitative analysis}

All the results from the IMI questionnaires are shown in fig. 2, in which we present the descriptive statistics in the upper panels to show the distribution of the data, and the results from the Friedman's tests with stepwise step-down analysis in the lower panels. Note that the order along the $\mathrm{x}$-axis was determined by the rank of each game, placing the highest ranked game for each subscale at the left, and 
the lowest ranked game at the right side to accommodate the visual representation of the homogeneous groups. Overall, the median IMI scores seem quite high, but some differentiations can be made.

Significant differences in ranking on the subscale enjoyment were found, $X^{2}(7)=49.516, p<.001$. Post hoc analysis show that especially the games Adventure, Wiiyoga, Kinski and Boxing had high rankings and appeared in the highest ranked homogeneous subset.

On the subscale effort, significant differences were found, $X^{2}(7)=18.865, p=.009$. However, all games, except the Cityride game were part of the highest ranked homogeneous subset.

Significant differences in ranks were also found in the subscale value, $X^{2}(7)=49.304, p<.001$. Wiiyoga distinguished itself from all other games, showing up singled out in the highest ranked homogeneous subset. The second homogeneous subset contained all other games except for Reach the Sky.

The subscale competence also resulted in significant differences, $X^{2}(7)=81.035, p<.001$. Three games, Wii yoga, Adventure and Boxing, formed the highest ranked homogeneous subset, which were all three also in the highest ranks for the subscale interest.

Finally significant differences in ranks were found in the subscale tension, $X^{2}(7)=31.631, p<.001$. The games Wiiski, Kinyoga, Kinski and Cityride form the highest ranked homogeneous subset. The three games that were in the highest ranked subset on both competence and interest ended up as the lowest three for the subscale tension. 


\section{Qualitative analysis}

To understand why older adults preferred certain games, we analyzed the Means-End-Chains (MECs) as obtained through UX-laddering and preference ranking. We ranked the games according to the preferences, in which second and favorite games, received one and two points respectively. The top three games were Adventure, Kinyoga and Boxing (fig. 3).

From the interviews 859 data-points were obtained from 30 participants, which formed 224 ladders. We differentiated 17 different attributes, 7 consequences and 7 values. We will highlight a few key MECs, a comprehensive overview of the links can be deducted from the HVM (fig. 4).

MEC1 - Variation - Physically active - Health and independence

The consequence 'Physically active' plays a central role as shown by the multiple links. Especially the link between 'Physically active' and the attribute 'Variation' is strong. 'Physically active' is further connected to the values, 'Health and Independency', 'Affinity', 'Motivating and Improve Fitness'. Participants often mentioned phrases like: 'The game was very active because I had to duck and jump, "I want to remain independent" and "I want to able to do the things I used to do".

MEC2 - Visual feedback - Positive feedback - Drive to perform

Another important value is 'Drive to Perform', which is linked to 'Visual Feedback', and 'Positive Feedback'. Participants would mention phrases such as: "I liked it because I received a good score" and "It feels good to perform well".

The value Affinity was mentioned in ladders that started from the attribute 'Realistic'. Participants mentioned things like: "I used to enjoy this activity" when they recognized things like skiing, driving a car or even boxing.

\section{Discussion}

The value of VR games

The IMI scores on the games were quite high, indicating that our participants were motivated by playing VR balance games. Furthermore, scores on the subscale enjoyment, which is considered the subscale that represents self-reported intrinsic motivation, ${ }^{19}$ were high. Furthermore, most games show high scores on the subscale value, indicating that older adults believe that these games can be effective. In 
this subscale, the Wiiyoga game distinguished itself from the other games. Possibly, because this game included important attributes like 'Visual Feedback' and is 'Physically Active', which helps to 'Improve Fitness' (fig. 4). Furthermore, the high score of this game on the subscale effort shows that older adults were motivated to put effort into the games. However, in previous work in which the challenge of VR games was studies by analyzing muscular activity, or by analyzing weight shifts, we have shown that very little muscular effort is needed to perform any of these games, ${ }^{28}$ and games that targeted weight shifts leave room for improvement. ${ }^{29}$ The high scores on the subscale effort are possibly due to a high cognitive challenge, after all 'Stay Focused' was mentioned as an important value. However, cognitive challenge in VR training is hard to measure and has not yet been studied as far as we know.

Important game mechanics

Remarkable are the conflicting results of the yoga games when comparing the preference ranking with the IMI results. Kinyoga ranked second best on the preference ranking (fig. 3), whereas it is badly ranked in the IMI subscales tension, enjoyment and competence. We speculate that this might be because Kinyoga looks attractive on a screenshot, as it clearly shows the potentially valuable 'visual feedback'. However, the feedback was often inconsistent resulting in frustration and low scores. Furthermore, the laddering was done at the end of the experiment, when the negative experience might have diminished. The values on the HVM show the importance of 'fitness and health' for older adults. These values are linked to the game mechanics 'Physically Active' and 'Variation'. Even though, incorporating variation might increase complexity, variation was shown to be an important factor in this study and in previous studies. ${ }^{30}$ A preference for 'variation' in movements as well as in the game environment was shown. These game mechanics, should not be neglected when developing VR-training games for older adults. The value 'Drive to Perform', which refers to the performance, or score in the game also shows to be an important value. This is in line with previous findings that show challenge, described as: "To push oneself to beat the game.", is an important motivator for older adults. ${ }^{14}$ The value 'Drive to perform' is linked to 'visual feedback', and 'positive feedback'. Adventure and Wiiyoga stood out by being the highest ranked games in all, but the negative subscale tension. These games are very different games, but are similar in the fact that they both show clear positive feedback, although for the Adventure game this does not show on the HVM. 


\section{Considerations for the older gamer}

A study by Broady et al. concluded that older adults can learn to use technology in the same ways as young, but emphasis should be on time to familiarize and on positive feedback to avoid fear of technology. ${ }^{31}$ Furthermore, as age increased an increase in enjoyment, effort and tension was observed, whereas competence declined. ${ }^{31}$ The results of our laddering study also indicate the importance of presenting 'slow movements', which helps to 'stay relaxed,' as can be seen in the HVM. All of the scores on the tension subscale were below the neutral score of three, indicating that little pressure was experienced. The games in the highest group of the subscale tension were games in which we often observed difficulties with the controls and the speed of the game. Moreover, games that induced high tension were games that scored low on the positive subscales.

Finally, some contradicting key elements show up on the HVM, such as 'Slow Movements', and 'Speed' (fig. 4). It is clear that these attributes lead to different consequences and values. When considering these attributes and their links together, it can be concluded that game speed should be fast enough to increase challenge, but not so fast, that it compromises the value 'Stay Relaxed'. This emphasizes the importance of games to be adaptable to the skills of the player.

\section{Limitations}

The high IMI scores obtained in this study, might be attributable to a selection bias. Participants volunteered which might result in a sample that holds positive views towards computer use and VR. Additionally, some participants were recruited around sport facilities so they will be inclined to belief that physical activity is important. However, the repeated measures design enables us to differentiate between games.

The variation of game types, and thus attributes, we introduced were limited because we exclusively tested readily available VR games that were controlled with body movements. Therefore, some attributes that are motivating for older adults, might not be present in our HVM. For example, older players tend to prefer completion, choice and enjoyment, ${ }^{32}$ which are aspects of exploration type games which were not among the included games. The games were also played individually, which eliminated the impact of social interaction. Social interaction was shown to be an important element that correlated with longer playing time in older players. ${ }^{14}$ Accordingly, further research is needed after adapting VR balance training for older adults to the knowledge that has been gained through recent research. This will lead to an evolution in understanding goal directed and population specific preferences in VR training. 


\section{Conclusions}

We have shown that VR training can lead to strong intrinsic motivation, overall older adults showed high enjoyment of VR based training games. Further dissecting what may explain high enjoyment, especially games that provide 'positive feedback' resulted in high IMI scores. 'Health and Independency' and 'Improve Fitness' were important values for older adults, connected to game mechanics like 'Variation' and 'Physically Active'. Furthermore, the 'Drive to Perform' was important for our participants, which was enhanced by 'Visual Feedback' and 'Positive Feedback'. Contradictory favorable attributes such as 'Speed' on one hand and 'Slow Movements' on the other hand, emphasize the importance of designing VR training that is adaptable to the skill level of the player. When designing or selecting VR-balancetraining games for older adults, these attributes should be considered to optimize user experience, thereby increasing intrinsic motivation. Novel games will have to go through further user experience analysis, to gain more detailed information about game mechanics that can increase motivation and finally improve adherence to VR-balance-training programs.

\section{Acknowledgements}

This research was funded by the European Commission through MOVE-AGE, an Erasmus Mundus Joint Doctorate programme (2011-0015).

\section{Conflict of interest}

We can confirm that no competing financial interests exist.

\section{e-mail:}

Corresponging author: Prof. dr. Sabine M.P. Verschueren: sabine.verschueren@kuleuven.be First author: Aijse de Vries: aijse.devries@kuleuven.be

\section{References}

1. Kannus P, Uusi-Rasi K, Palvanen M, et al. Non-pharmacological means to prevent fractures among older adults. Ann Med. 2005;37(4):303-310.

2. Moreland JD, Richardson JA, Goldsmith CH, et al. Muscle Weakness and Falls in Older Adults: A Systematic Review and Meta-Analysis. J Am Geriatr Soc. 2004;52(7):1121-1129.

3. van Diest M, Lamoth CJC, Stegenga J, et al. Exergaming for balance training of elderly: state of the art and future developments. J Neuroeng Rehabil. 2013;10:101.

4. Goble DJ, Cone BL, Fling BW. Using the Wii Fit as a tool for balance assessment and neurorehabilitation: the first half decade of "Wii-search." J Neuroeng Rehabil. 2014;11(1):12-.

5. Donath L, Rössler R, Faude O. Effects of Virtual Reality Training (Exergaming) Compared to Alternative Exercise Training and Passive Control on Standing Balance and Functional Mobility in Healthy Community-Dwelling Seniors: A Meta- 
Analytical Review. Sport Med. 2016:1-17.

6. Wollersheim D, Merkes M, Shields N, et al. Physical and Psychosocial Effects of Wii Video Game Use among Older Women. Int J Emerg Technol Soc. 2010;8(2):85-98.

7. Sherrington C, Tiedemann A, Fairhall N, et al. Exercise to prevent falls in older adults: an updated meta-analysis and best practice recommendations. N S W Public Heal Bull. 2011;22(3-4):78-83.

8. Granacher U, Muehlbauer T, Gruber M. A qualitative review of balance and strength performance in healthy older adults: Impact for testing and training. J Aging Res. 2012;2012.

9. Stineman MG, Strumpf N, Kurichi JE, et al. Attempts to Reach the Oldest and Frailest: Recruitment, Adherence, and Retention of Urban Elderly Persons to a Falls Reduction Exercise Program. Gerontologist. 2011;51(suppl_1):S59-S72.

10. Duque G, Boersma D, Loza-Diaz G, et al. Effects of balance training using a virtual-reality system in older fallers. Clin Interv Aging. 2013;8:257-263.

11. Kramer A, Dettmers C, Gruber M. Exergaming With Additional Postural Demands Improves Balance and Gait in Patients With Multiple Sclerosis as Much as Conventional Balance Training and Leads to High Adherence to Home-Based Balance Training. Arch Phys Med Rehabil. 2014;95(10):1803-1809.

12. Milgram P. Augmented reality: A class of displays on the reality-virtuality continuum. Telemanipulator Telepresence Technol. 1994;2351:282-292.

13. Nawaz A, Skjæret N, Helbostad JL, et al. Usability and acceptability of balance exergames in older adults: A scoping review. Health Informatics J. 2016;22(4):911-931.

14. De Schutter B. Never Too Old to Play: The Appeal of Digital Games to an Older Audience. Games Cult. 2010;6(2):155170.

15. Birk M V., Atkins C, Bowey JT, et al. Fostering Intrinsic Motivation through Avatar Identification in Digital Games. In: Proceedings of the 2016 CHI Conference on Human Factors in Computing Systems - CHI '16. ; 2016:2982-2995.

16. Ryan RM, Mims V, Koestner R. Relation of reward contingency and interpersonal context to intrinsic motivation: $A$ review and test using cognitive evaluation theory. J Pers Soc Psychol. 1983;45(4):736-750.

17. Mandryk RL, Inkpen KM, Calvert TW. Using psychophysiological techniques to measure user experience with entertainment technologies. Behav Inf Technol. 2006;25(2):141-158.

18. Ryan RM. Control and information in the intrapersonal sphere: An extension of cognitive evaluation theory. $J$ Pers Soc Psychol. 1982;43(3):450-461.

19. Deci EL, Ryan RM. (2003). Intrinsic motivation inventory. Self-Determination Ther. 2003;267.

20. Fitzgerald D, Trakarnratanakul N, Smyth B, et al. Effects of a wobble board-based therapeutic exergaming system for balance training on dynamic postural stability and intrinsic motivation levels. J Orthop Sports Phys Ther. 2010;40(1):1119.

21. Pieters R, Baumgartner H, Allen D. A means-end chain approach to consumer goal structures. Int J Res Mark. $1995 ; 12(3): 227-244$.

22. Gutman J. A Means-End Chain Model Based on Consumer Categorization Processes. J Mark. 1982;46(2):60-72.

23. Reynolds TJ, Gutman J. Laddering theory, method, analysis, and interpretation. J Advert Res. 1988;28(1):11-31.

24. Vanden Abeele V, Zaman B, De Grooff D. User eXperience Laddering with preschoolers: unveiling attributes and benefits of cuddly toy interfaces. Pers Ubiquitous Comput. 2012;16(4):451-465.

25. Galea M, Woodward M. Mini-mental state examination (MMSE). Aust J Physiother. 2005;51(3):198. 
26. Urbaniak C G, Plous S. Research Randomizer (Version 4.0) [Computer software]. 2013. http://www.randomizer.org/.

27. LadderUX on line and freely available software tool for UX laddering. www.ladderux.org.

28. de Vries AW, van Dieën JH, Jonkers I, et al. Virtual Reality Balance Games Provide Little Muscular Challenge to Prevent Muscle Weakness in Healthy Elderly. Clin Biomech. Under Rev.

29. de Vries AW, Faber G, Jonkers I, et al. Virtual reality balance training for elderly: Similar skiing games elicit different challenges in balance training. Gait Posture. 2018;59.

30. Skjaeret N, Nawaz A, Ystmark K, et al. Designing for Movement Quality in Exergames: Lessons Learned from Observing Senior Citizens Playing Stepping Games. Gerontology. 2015;61(2):186-194.

31. Broady T, Chan A, Caputi P. et al. Comparison of older and younger adults' attitudes towards and abilities with computers: Implications for training and learning. Br J Educ Technol. 2010;41(3):473-485.

32. Birk M V, Friehs MA, Mandryk RL. Age-Based Preferences and Player Experience: A Crowdsourced Cross-sectional Study. In: Proceedings of the Annual Symposium on Computer-Human Interaction in Play. ACM; 2017:157-170. 